\title{
PROBLEMAS COM O USO DE PROGRAMAÇÃO LINEAR COM POSTERIOR ARREDONDAMENTO DA SOLUÇÃO ÓTIMA, EM REGULAÇÃO FLORESTAL ${ }^{1}$
}

\author{
Gilson Fernandes da Silva², Helio Garcia Leite ${ }^{3}$, Márcio Lopes da Silva ${ }^{3}$, Flávio Lopes Rodrigues² e \\ Heleno do Nascimento Santos ${ }^{4}$
}

\begin{abstract}
RESUMO - O principal objetivo deste trabalho foi avaliar, com a ajuda da Programação Inteira, as conseqüências do uso da Programação Linear com posterior arredondamento das respostas, com ênfase na regulação de florestas eqüiâneas. Para isto, um problema de regulação florestal simplificado foi idealizado e resolvido pelo modelo I, por meio de Programação Linear, Programação Linear com posterior arredondamento e Programação Inteira. Ao final, concluiu-se que o arredondamento das respostas obtidas pelo modelo resolvido por Programação Linear levou a uma solução inviável para o problema de regulação proposto. O mesmo não ocorreu com o modelo de Programação Inteira, que apresentou um plano de regulação factível e otimizado, mostrando que, do ponto de vista matemático, respostas com arredondamento da solução de modelos de Programação Linear não devem ser adotadas.
\end{abstract}

Palavras-chave: Programação Linear, Programação Inteira e manejo florestal.

\section{PROBLEMS USING LINEAR PROGRAMMING WITH A POST ROUNDING OUT OF THE OPTIMAL SOLUTION IN FOREST REGULATION}

\begin{abstract}
The main objective of this work was to evaluate, through Integer Programming, the consequences of using Linear Programming with post rounding out of the responses, with emphasis on even-aged forest regulation. Thus, a simplified forest regulation problem was proposed out and solved by model I by means of Linear Programming, Linear Programming with post rounding out, and Integer Programming. It was concluded that the rounding out of responses obtained by the model solved by Linear Programming led to an unviable solution for the proposed regulation problem. The same did not occur with the Integer Programming model, which presented a viable, optimal regulation plan, showing that, from a mathematical viewpoint, responses with rounding out of solution using Linear Programming models should not be adopted.
\end{abstract}

Key words: $\quad$ Linear Programming, Integer Programming and forest regulation.

\section{INTRODUÇÃO}

De acordo com Rodriguez \& Lima (1985) e Leite (1994), entre as técnicas de Pesquisa Operacional aplicadas ao planejamento florestal, a Programação Linear aparece como uma das principais. Uma das pressuposições desse tipo de Programação Matemática, conforme Hiller \& Lieberman (1980), é a divisibilidade, ou seja, a pressuposição de que toda variável de decisão pode assumir qualquer valor real não-negativo (inteiro ou

1 Recebido para publicação em 16.5.2002.

Aceito para publicação em 9.9.2003.

2 Professor do Centro de Ciências Agrárias da Universidade Federal do Espírito Santo, Alto Universitário, Caixa Postal 16, 29500-000 Alegre-ES, <gfsilva2000@yahoo.com>. ${ }^{3}$ Professor do Departamento de Engenharia Florestal da Universidade Federal de Viçosa - UFV, 36571-000 Viçosa-MG; ${ }^{4}$ Professor do Departamento de Informática da UFV. 
fracionário). Em muitas situações do planejamento florestal, essa divisibilidade pode resultar na impossibilidade de adoção da solução ótima, principalmente se as variáveis decisórias envolverem mão-de-obra ou máquinas, e, também, em degeneração do ótimo, quando são adotadas soluções operacionais. Uma solução operacional consiste em uma aproximação exeqüível do ótimo indicado pela Programação Matemática.

Considerando um modelo de planejamento florestal em que as variáveis de decisão representam áreas de unidades de manejo a serem submetidas a um determinado regime de manejo, é comum encontrar como solução para estes modelos subdivisões dessas unidades entre diferentes regimes de manejo. Essas soluções são em geral indesejáveis do ponto de vista operacional, mas possíveis de ocorrer do ponto de vista matemático, pois trabalhase com variáveis definidas no espaço real de valores contínuos e positivos (Clutter et al., 1983; Dykstra, 1984; Davis \& Johnson, 1987).

Situações que envolvem variáveis inteiras são comuns no manejo florestal, justificando, portanto, o estudo de métodos de solução de problemas de Programação Matemática com restrições de integridade. Nobre (1999) sugeriu como solução para resolver o problema da divisão das unidades de manejo, em estudos de regulação florestal, o uso de Programação Inteira com variáveis dummy. Estas variáveis permitiriam, então, definir a associação ou não de um determinado regime de manejo com a respectiva unidade de produção. Contudo, de acordo com Dykstra (1984) e Buongiorno \& Gilless (1987), problemas com variáveis inteiras são difíceis de resolver, mesmo com computadores de grande desempenho. O que tem sido proposto e feito no Brasil para contornar essa situação é a solução de problemas de grande porte por meio do emprego da Programação Linear com posterior arredondamento das respostas, embora se saiba que outros algoritmos têm sido utilizados em pesquisas para resolver problemas que exigem a integridade das variáveis. Entre estes, as metas heurísticas têm sido alvo de vários estudos, dado ao seu grande desempenho computacional (Lockwood \& More 1993; Laroze \& Greber, 1997; Laroze, 1999; Boston \& Bettinger, 1999; Nobre, 1999; Rodrigues, 2001).

Embora o arredondamento de soluções muitas vezes possa parecer a saída mais fácil e prática para problemas de planejamento florestal de grande porte, conforme mencionado anteriormente, esta prática pode levar a resultados insatisfatórios e, muitas vezes, a soluções inviáveis. De acordo com Goldbarg \& Luna (2000), a técnica de arredondamento pode resultar em uma derrocada completa no esforço de modelagem e solução, o que justifica o uso de outros métodos de solução. Neste estudo, procurou-se avaliar, com a ajuda da Programação Inteira, as consequiências do uso da Programação Linear com posterior arredondamento das respostas, com ênfase na regulação de florestas equiiâneas.

\section{MATERIAL E MÉTODOS}

Com vistas a alcançar o objetivo proposto, foram utilizados dados de um problema de regulação florestal simplificado, mas que possui número suficiente de variáveis para ilustrar as situações desejadas. Esse problema consistiu em: uma empresa florestal deseja elaborar um plano de manejo para uma floresta eqüiânea para os próximos 8 anos (horizonte de planejamento), com o objetivo de maximizar o retorno econômico. Os dados desse exemplo estão apresentados no Quadro 1.

Quadro 1 - Áreas, idades atuais e produção das unidades de manejo

Table 1 - Areas, current ages and yield of the management units

\begin{tabular}{|c|c|c|c|}
\hline $\begin{array}{c}\text { Unidade de } \\
\text { Manejo }\end{array}$ & $\begin{array}{c}\text { Área } \\
\text { (ha) }\end{array}$ & Idade Atual & $\begin{array}{c}\text { Produção } \\
\left(\mathrm{m}^{3} / \mathrm{ha}\right)\end{array}$ \\
\hline 1 & 50,00 & 1 & \\
\hline 2 & 85,00 & 2 & \\
\hline 3 & 50,00 & 3 & \\
\hline 4 & 50,00 & 4 & 5 \\
\hline 5 & 90,00 & 5 & 179,10 \\
\hline 6 & 35,00 & 6 & 235,02 \\
\hline 7 & 35,00 & 7 & 285,37 \\
\hline 8 & 60,00 & 8 & 300,00 \\
\hline
\end{tabular}

São fornecidas também as seguintes informações:

- as possíveis atividades incluem: corte seguido de reforma imediata;

- as idades mínima e máxima de corte são 5 e 8 anos, respectivamente;

- as operações de corte ocorrem uma vez a cada ano, no início do ano;

- os sítios são homogêneos; e

- a demanda anual do mercado por madeira é de $15.000,00 \mathrm{~m}^{3} / \mathrm{ano}$. 


\subsection{Formulação pelo Modelo Clássico de Programação Linear (PL)}

Inicialmente o problema proposto foi formulado como um problema de Programação Linear clássico. Foi empregado o modelo I, proposto originalmente por Kidd et al. (1966), citados por Dykstra (1984), mas que recebeu essa denominação de Johnson \& Scheurman (1977). Nessa formulação, a variável de decisão $X_{i j}$ representa a fração da unidade de manejo $i$, que deverá ser assinalada na prescrição $j$. O fundamento básico dessa formulação é de que a floresta é subdividida em classes homogêneas de idade, sendo posteriormente prescrito um elenco predeterminado de alternativas de manejo para cada classe. As prescrições representam sequiências preestabelecidas de ações que ocorrerão ao longo do período de transição (Ribeiro, 1996). As prescrições podem se referir, por exemplo, às idades de colheita. No modelo I, uma vez que uma certa fração de área seja assinalada em uma determinada prescrição de manejo, ela permanecerá sob tal prescrição durante todo o horizonte de planejamento. Este horizonte é subdividido em períodos, e as ações ocorrem no início destes, conforme Ribeiro (1996).

Prescrições de manejo foram sugeridas para o problema em questão, conforme apresentado no Quadro 2, em que a variável de decisão $X_{i j}$ representa a área em hectares da unidade de manejo $i$, a qual será atribuída à prescrição $j$. Para determinação das prescrições, foram consideradas como idades de corte, mínima e máxima, 5 e 8 anos, respectivamente.

Considerou-se como objetivo do empreendedor a maximização do benefício periódico equivalente (BPE) global da floresta, sendo estabelecida a seguinte funçãoobjetivo:

$$
\operatorname{Max} \mathrm{BPE}_{G}=\sum_{i=1}^{M} \sum_{j=1}^{N} C_{i j} X_{i j}
$$

em que $B P E_{G}=$ benefício periódico equivalente (BPE) global; $C_{i j}=$ benefício periódico equivalente $(\mathrm{BPE}) \mathrm{da}$ exploração de cada hectare da unidade de manejo $i$, manejada sob a alternativa de manejo $j ; X_{i j}=$ fração de área (ha) da unidade de manejo $i$ assinalada na alternativa de manejo $j ; M=$ número total de unidades de manejos; e $N=$ número total de alternativas de manejo da i-ésima unidade de manejo.

Para obtenção do benefício periódico equivalente (BPE), empregou-se a metodologia apresentada em
Rezende \& Oliveira (1993) e Gomes et al. (1998). Deste modo, o BPE foi obtido pela seguinte expressão:

$$
B P E=\frac{V P L . i}{\left[1-(1+i)^{-n}\right]}
$$

em que $B P E=$ benefício periódico equivalente, em $\mathrm{R} \$$ / ha/ano; $V P L=$ valor presente líquido, em $\mathrm{R} \$ /$ ha; $i=$ taxa de desconto; e $n=$ duração do projeto, em anos.

Foram considerados uma taxa de desconto (i) de $7 \%$ a.a. e o preço da madeira posto na fábrica de $\mathrm{R} \$ 42,00 / \mathrm{m}^{3}$, em média. Os valores dos custos estão apresentados no Quadro 3.

A partir dos valores de custos e receitas apresentados, a função-objetivo assumiu a seguinte forma:

MAX Z $=177,58 X_{11}+341,03 X_{12}+471,75 X_{13}+158,50 X_{21}+$
$318,79 X_{22}+438,46 X_{23}+441,63 X_{24}+146,31 X_{31}+301,91 X_{32}$
$+420,69 X_{33}+415,93 X_{34}+514,75 X_{41}+292,26 X_{42}+408,02 X_{43}$
$+403,25 X_{44}+500,16 X_{51}+621,54 X_{52}+642,77 X_{53}+402,44 X_{54}$
$+394,53 X_{55}+496,41 X_{61}+613,19 X_{62}+706,58 X_{63}+634,78 X_{64}$
$+750,12 X_{65}+739,51 X_{66}+391,93 X_{67}+637,44 X_{71}+749,04 X_{72}$
$+838,29 X_{73}+738,18 X_{74}+848,42 X_{75}+716,40 X_{76}+747,23 X_{81}$
$+854,39 X_{82}+940,09 X_{83}+718,76 X_{84}+824,60 X_{85}$

Observe, por exemplo, que o valor 177,58 representa a receita líquida anual (lucro) obtida de cada hectare da unidade de manejo 1, segundo a alternativa de manejo 1 (corte aos cinco anos de idade).

\subsubsection{Restrição de área disponível para colheita}

Esta é a restrição que determina a disponibilidade total de área de cada unidade de manejo florestal, sendo:

$$
\sum_{i=1}^{M} \sum_{j=1}^{N} X_{i j}=A_{i}(\mathrm{i}=1, \ldots, \mathrm{M} \mathrm{e} \mathrm{j}=1, \ldots, \mathrm{N})
$$

em que $A_{i}=$ área da i-ésima unidade de manejo.

Para o exemplo, esta restrição pode ser escrita da seguinte forma:

$\mathrm{X}_{11}+\mathrm{X}_{12}+\mathrm{X}_{13} \leq 50$

$\mathrm{X}_{21}+\mathrm{X}_{22}+\mathrm{X}_{23}+\mathrm{X}_{24} \leq 85$

$\mathrm{X}_{31}+\mathrm{X}_{32}+\mathrm{X}_{33}+\mathrm{X}_{34} \leq 50$

$X_{41}+X_{42}+X_{43}+X_{44} \leq 50$

$\mathrm{X}_{51}+\mathrm{X}_{52}+\mathrm{X}_{53}+\mathrm{X}_{54}+\mathrm{X}_{55} \leq 90$

$\mathrm{X}_{61}+\mathrm{X}_{62}+\mathrm{X}_{63}+\mathrm{X}_{64}+\mathrm{X}_{65}+\mathrm{X}_{66}+\mathrm{X}_{67} \leq 35$

$X_{71}+X_{72}+X_{73}+X_{74}+X_{75}+X_{76} \leq 35$

R. Árvore, Viçosa-MG, v.27, n.5, p.677-688, 2003 
Quadro 2 - Prescrições viáveis de manejo para um horizonte de planejamento de 8 anos

Table 2 - Viable management prescriptions for an 8-year horizon planning

\begin{tabular}{|c|c|c|c|c|c|c|c|c|c|c|c|}
\hline \multirow{2}{*}{ Prescrição } & \multirow{2}{*}{$\begin{array}{l}\text { Idade } \\
\text { Atual }\end{array}$} & \multirow{2}{*}{ Rotação } & \multicolumn{8}{|c|}{ Horizonte de Planejamento } & \multirow{2}{*}{$\begin{array}{c}\text { Idade } \\
\text { Fina }\end{array}$} \\
\hline & & & 0 & 1 & 2 & 3 & 4 & 5 & 6 & 7 & \\
\hline $\mathrm{X}_{11}$ & 0 & 5 & & & & & & 179,10 & & & 3 \\
\hline $\mathrm{X}_{12}$ & 0 & 6 & & & & & & & 235,02 & & 2 \\
\hline $\mathrm{X}_{13}$ & 0 & 7 & & & & & & & & 285,37 & 1 \\
\hline$X_{21}$ & 1 & 5 & & & & & 179,10 & & & & 4 \\
\hline$X_{22}$ & 1 & 6 & & & & & & 235,02 & & & 3 \\
\hline$X_{23}$ & 1 & 7 & & & & & & & 285,37 & & 2 \\
\hline $\mathrm{X}_{24}$ & 1 & 8 & & & & & & & & 300,00 & 1 \\
\hline $\mathrm{X}_{31}$ & 2 & 5 & & & & 179,10 & & & & & 5 \\
\hline $\mathrm{X}_{32}$ & 2 & 6 & & & & & 235,02 & & & & 4 \\
\hline$X_{33}$ & 2 & 7 & & & & & & 285,37 & & & 3 \\
\hline $\mathrm{X}_{34}$ & 2 & 8 & & & & & & & 300,00 & & 2 \\
\hline $\mathrm{X}_{41}$ & 3 & $5-5$ & & & 179,10 & & & & & 179,10 & 1 \\
\hline$X_{42}$ & 3 & 6 & & & & 235,02 & & & & & 5 \\
\hline $\mathrm{X}_{43}$ & 3 & 7 & & & & & 285,37 & & & & 4 \\
\hline $\mathrm{X}_{44}$ & 3 & 8 & & & & & & 300,00 & & & 3 \\
\hline $\mathrm{X}_{51}$ & 4 & $5-5$ & & 179,10 & & & & & 179,10 & & 2 \\
\hline $\mathrm{X}_{52}$ & 4 & $5-6$ & & 179,10 & & & & & & 235,02 & 1 \\
\hline $\mathrm{X}_{53}$ & 4 & $6-5$ & & & 235,02 & & & & & 179,10 & 1 \\
\hline $\mathrm{X}_{54}$ & 4 & 7 & & & & 285,37 & & & & & 5 \\
\hline $\mathrm{X}_{55}$ & 4 & 8 & & & & & 300,00 & & & & 4 \\
\hline $\mathrm{X}_{61}$ & 5 & $5-5$ & 179,10 & & & & & 179,10 & & & 3 \\
\hline $\mathrm{X}_{62}$ & 5 & $5-6$ & 179,10 & & & & & & 235,02 & & 2 \\
\hline$X_{63}$ & 5 & $5-7$ & 179,10 & & & & & & & 285,37 & 1 \\
\hline $\mathrm{X}_{64}$ & 5 & $6-5$ & & 235,02 & & & & & 179,10 & & 2 \\
\hline$X_{65}$ & 5 & $6-6$ & & 235,02 & & & & & & 235,02 & 1 \\
\hline $\mathrm{X}_{66}$ & 5 & $7-5$ & & & 285,37 & & & & & 179,10 & 1 \\
\hline $\mathrm{X}_{67}$ & 5 & 8 & & & & 300,00 & & & & & 5 \\
\hline $\mathrm{X}_{71}$ & 6 & $6-5$ & 235,02 & & & & & 179,10 & & & 3 \\
\hline$X_{72}$ & 6 & $6-6$ & 235,02 & & & & & & 235,02 & & 2 \\
\hline$X_{73}$ & 6 & $6-7$ & 235,02 & & & & & & & 285,37 & 1 \\
\hline$X_{74}$ & 6 & $7-5$ & & 285,37 & & & & & 179,10 & & 2 \\
\hline $\mathrm{X}_{75}$ & 6 & $7-6$ & & 285,37 & & & & & & 235,02 & 1 \\
\hline $\mathrm{X}_{76}$ & 6 & $8-5$ & & & 300,00 & & & & & 179,10 & 1 \\
\hline $\mathrm{X}_{81}$ & 7 & $7-5$ & 285,37 & & & & & 179,10 & & & 3 \\
\hline $\mathrm{X}_{82}$ & 7 & $7-6$ & 285,37 & & & & & & 235,02 & & 2 \\
\hline$X_{83}$ & 7 & $7-7$ & 285,37 & & & & & & & 285,37 & 1 \\
\hline $\mathrm{X}_{84}$ & 7 & $8-5$ & & 300,00 & & & & & 179,10 & & 2 \\
\hline $\mathrm{X}_{85}$ & 7 & $8-6$ & & 300,00 & & & & & & 235,02 & 1 \\
\hline & \multicolumn{2}{|c|}{ Estoque de colheita } & & & & & & & & & \\
\hline & \multicolumn{2}{|c|}{ Estoque de crescimento } & & & & & & & & & \\
\hline
\end{tabular}


Quadro 3 - Custos médios das principais atividades

Table 3 -Average costs of the main activities

\begin{tabular}{|c|c|c|}
\hline Atividade & Custo (R\$/ha) & Ano de Ocorrência \\
\hline $\begin{array}{l}\text { Implantação } \\
\text { Manutenção } \\
\text { Reforma } \\
\text { Exploração }\left(\mathrm{R} \$ / \mathrm{m}^{3}\right) \\
\text { Custo anual da terra }\end{array}$ & $\begin{array}{r}1.040,12 \\
97,12 \\
168,85 \\
79,65 \\
79,65 \\
40,25 \\
140,13 \\
140,13 \\
871,27 \\
10,91 \\
1.200 \times \text { i }\end{array}$ & $\begin{array}{c}0 \\
0 \\
\text { No ano } 1 \text { e no } 1^{\circ} \text { ano após reforma } \\
\text { No ano } 2 \text { e no } 2^{\circ} \text { ano após reforma } \\
\text { No ano } 3 \text { e no } 3^{\circ} \text { ano após reforma } \\
\text { Do } 4^{\circ} \text { até o ano do } 1^{\circ} \text { corte, o mesmo após reforma } \\
\text { No ano do } 1^{\circ} \text { corte } \\
\text { No ano do } 2^{\circ} \text { corte } \\
\text { Ano zero do } 2^{\circ} \text { ciclo } \\
\text { Nos anos do } 1^{\circ} \text { e } 2^{-o} \text { corte } \\
\text { Anualmente }\end{array}$ \\
\hline
\end{tabular}

\subsubsection{Imposição de Cotas ou Produções Anuais}

Esta restrição visa estabelecer uma produção periódica de volume de madeira de modo que atenda a uma demanda preestabelecida. Considerando-se $V_{k}$ o volume total removido no período $k$, então:

$$
V_{k}=\sum_{i=1}^{M} \sum_{j=1}^{N} V_{i j k} X_{i j}\{k=0,1, \ldots, H-2\}
$$

em que $V_{i j k}=$ volume $\left(\mathrm{m}^{3} / \mathrm{ha}\right)$ produzido pela i-ésima unidade de manejo assinalada na j-ésima alternativa de manejo, no início do período $k ; V_{k}=$ volume total $\left(\mathrm{m}^{3}\right)$ removido no período $k$, representando a demanda a ser atendida no período $k$; e $H=$ horizonte de planejamento.

Para o problema proposto, a demanda mínima do mercado por madeira é de $15.000,00 \mathrm{~m}^{3} /$ ano. Contudo, deseja-se também que a produção de madeira não exceda os 20.000,00 m³/ano, prevendo-se um excesso de produção, o que poderia acarretar dificuldades na sua estocagem e venda. Essas restrições podem ser assim representadas:

V0 : $20000 \geq 179,10 \mathrm{X}_{61}+179,10 \mathrm{X}_{62}+179,10 \mathrm{X}_{63}+$ $235,02 X_{71}+235,02 X_{72}+235,02 X_{73}+285,37 X_{81}+$ $285,37 X_{82}+285,37 X_{83} \geq 15000$

$\mathrm{V} 1: 20000 \geq 179,10 \mathrm{X}_{51}+179,10 \mathrm{X}_{52}+235,02 \mathrm{X}_{64}+$ $235,02 X_{65}+285,37 X_{74}+285,37 X_{75}+300,00 X_{84}+$ $300,00 \mathrm{X}_{85} \geq 15000$

$$
\begin{aligned}
& \text { V2: } 20000 \geq 179,10 X_{41}+235,02 X_{53}+285,37 X_{66}+ \\
& \text { 300,00 } X_{76} \geq 15000 \\
& \text { V3: } 20000 \geq 179,10 X_{31}+235,02 X_{42}+285,37 X_{54}+ \\
& \text { 300,00 } X_{67} \geq 15000 \\
& \text { V4: } 20000 \geq 179,10 X_{21}+235,02 X_{32}+285,37 X_{43}+ \\
& \text { 300,00 } X_{55} \geq 15000 \\
& \text { V5: } 20000 \geq 179,10 X_{11}+235,02 X_{22}+285,37 X_{33}+ \\
& 300,00 X_{44}+179,10 X_{61}+179,10 X_{71}+179,10 X_{81} \geq \\
& 15000 \\
& \text { V6 : } 20000 \geq 235,02 X_{12}+285,37 X_{23}+300,00 X_{34}+ \\
& 179,10 X_{51}+235,02 X_{62}+179,10 X_{64}+235,02 X_{72}+ \\
& 179,10 X_{74}+235,02 X_{82}+179,10 X_{84} \geq 15000 \\
& \text { V7: } 20000 \geq 285,37 X_{13}+300,00 X_{24}+179,10 X_{41}+ \\
& 235,02 X_{52}+179,10 X_{53}+285,37 X_{63}+235,02 X_{65}+ \\
& 179,10 X_{66}+285,37 X_{73}+235,02 X_{75}+179,10 X_{76}+ \\
& 285,37 X_{83}+235,02 X_{85} \geq 15000
\end{aligned}
$$

\subsection{Modelo de Programação Linear com Posterior Arredondamento}

O modelo de Programação Linear com posterior arredondamento é uma adaptação do modelo de Programação Linear muito utilizada por empresas, em que a solução ótima obtida pelo modelo de programação linear é arredondada para manter a integridade da unidade de manejo, levando-se em conta a alternativa de manejo que apresentou maior resposta. Este tipo de procedimento poderá ser mais bem visualizado nos Resultados e Discussões.

R. Árvore, Viçosa-MG, v.27, n.5, p.677-688, 2003 
Contudo, para melhor compreensão do leitor, considere o caso do modelo de Programação Linear apresentado no item anterior, em que a variável de decisão escolhida foi $X_{i j}$, que representa a fração de área (ha) da unidade de manejo $i$ assinalada na alternativa de manejo $j$. Assim, para ilustrar a aplicação do arredondamento da solução do resultado do modelo de Programação Linear, considere o seguinte exemplo: Assumindo-se que, de acordo com o modelo de Programação Linear proposto anteriormente, a unidade de manejo 1 pode ter três alternativas de manejo (corte aos 5, 6 e 7 anos), imagine que a resposta encontrada após a otimização do modelo foi $X_{11}=24, X_{12}=26$ e $X_{13}=0$. Interpretando esta resposta, significaria dizer que a unidade de manejo 1 , que tem uma área total de 50 ha, deveria ser subdividida em novas duas unidades, uma com 24 ha (variável $X_{l l}=24$ ) e outra com 26 ha (variável $X_{12}=26$ ). Para evitar esta subdivisão, pode-se fazer $o$ arredondamento da resposta, mantendose a unidade original íntegra em termos de alternativa de manejo, adotando-se aquela alternativa que apresentou maior resposta (variável $X_{12}=26$ ). Neste caso, o manejador em vez de dividir uma unidade de 50 ha em duas, uma com 24 e outra com 26 ha, pode decidir manter uma única unidade de manejo com os 50 ha originais, optando pela alternativa de manejo da maior resposta, ou seja, no caso a alternativa de manejo correspondente à variável $X_{12}$ com 26 ha, que indica corte aos 6 anos de idade. Assim, mantería-se uma única unidade de manejo cortada aos 6 anos de idade em vez de duas, cortadas aos 5 e 6 anos, respectivamente.

\subsection{Modelo de Programação Inteira (PI)}

Considerando agora $W_{i j}$ uma variável binária, em que 1 significa cortar a unidade de manejo $i$ assinalada na alternativa $j$ e 0 significa o contrário, foi estabelecida a seguinte função-objetivo:

$$
\text { Max. } B P E_{G}=\sum_{i=1}^{M} \sum_{j=1}^{N} b_{i j} W_{i j}
$$

em que $B P E_{G}=$ benefício periódico equivalente (BPE) global; $b_{i j}=$ benefício periódico equivalente (BPE) total de cada unidade de manejo $i$ assinalada na alternativa $j$; $M=$ número total de unidades de manejos; e $N=$ número total de alternativas de manejo da i-ésima unidade de manejo.

Substituindo-se os valores de $B P E$, obtidos conforme metodologia apresentada no item 2.1, multiplicados pelas respectivas áreas de cada unidade de manejo, a função-objetivo assumiu a seguinte forma:

MAX Z $=8878,97 \mathrm{~W}_{11}+17051,27 \mathrm{~W}_{12}+23587,33 \mathrm{~W}_{13}+$ $13472,87 \mathrm{~W}_{21}+27097,23 \mathrm{~W}_{22}+37269,04 \mathrm{~W}_{23}+37538,72 \mathrm{~W}_{24}$ $+7315,39 \mathrm{~W}_{31}+15095,39 \mathrm{~W}_{32}+21034,26 \mathrm{~W}_{33}+20796,27$ $\mathrm{W}_{34}+25737,34 \mathrm{~W}_{41}+14612,79 \mathrm{~W}_{42}+20401,17 \mathrm{~W}_{43}+$ $20162,66 \mathrm{~W}_{44}+45014,81 \mathrm{~W}_{51}+55938,65 \mathrm{~W}_{52}+57849,28 \mathrm{~W}_{53}$ $+36219,35 \mathrm{~W}_{54}+35507,62 \mathrm{~W}_{55}+17374,36 \mathrm{~W}_{61}+$ $21461,57 \mathrm{~W}_{62}+24730,45 \mathrm{~W}_{63}+22217,13 \mathrm{~W}_{64}+26254,36 \mathrm{~W}_{65}$ $+25882,84 \mathrm{~W}_{66}+13717,48 \mathrm{~W}_{67}+22310,28 \mathrm{~W}_{71}+$ $26216,25 \mathrm{~W}_{72}+29340,17 \mathrm{~W}_{73}+25836,35 \mathrm{~W}_{74}+29694,55$ $\mathrm{W}_{75}+25074,07 \mathrm{~W}_{76}+26153,14 \mathrm{~W}_{81}+29903,67 \mathrm{~W}_{82}+$ $32903,27 \mathrm{~W}_{83}+25156,50 \mathrm{~W}_{84}+28861,17 \mathrm{~W}_{85}$

\subsubsection{Restrição de Área Disponível para Colheita}

Esta restrição determina se toda a área da unidade de manejo florestal será cortada ou não, sendo:

$$
\sum_{i=1}^{M} \sum_{j=1}^{N} W_{i j}=A_{i}(\mathrm{i}=1, \ldots, \mathrm{Mej}=1, \ldots, \mathrm{N})
$$

$A_{i}$ é sempre igual a 1 e $W_{i j} \in\{0,1\}$ em que $0=$ não-assinalamento da alternativa $\mathrm{j}$ na unidade de manejo i; e 1 = caso contrário.

Essa restrição pode ser escrita, ainda, da seguinte forma:

$$
\begin{aligned}
& \mathrm{W}_{11}+\mathrm{W}_{12}+\mathrm{W}_{13}=1 \\
& \mathrm{~W}_{21}+\mathrm{W}_{22}+\mathrm{W}_{23}+\mathrm{W}_{24}=1 \\
& \mathrm{~W}_{31}+\mathrm{W}_{32}+\mathrm{W}_{33}+\mathrm{W}_{34}=1 \\
& \mathrm{~W}_{41}+\mathrm{W}_{42}+\mathrm{W}_{43}+\mathrm{W}_{44}=1 \\
& \mathrm{~W}_{51}+\mathrm{W}_{52}+\mathrm{W}_{53}+\mathrm{W}_{54}+\mathrm{W}_{55}=1 \\
& \mathrm{~W}_{61}+\mathrm{W}_{62}+\mathrm{W}_{63}+\mathrm{W}_{64}+\mathrm{W}_{65}+\mathrm{W}_{66}+\mathrm{W}_{67}=1 \\
& \mathrm{~W}_{71}+\mathrm{W}_{72}+\mathrm{W}_{73}+\mathrm{W}_{74}+\mathrm{W}_{75}+\mathrm{W}_{76}=1 \\
& \mathrm{~W}_{81}+\mathrm{W}_{82}+\mathrm{W}_{83}+\mathrm{W}_{84}+\mathrm{W}_{85}=1
\end{aligned}
$$

\subsubsection{Imposição de Cotas ou Produções Anuais}

Da mesma forma que no modelo de Programação Linear, para uma demanda mínima de 15.000,00 m³/ano e máxima de 20.000,00 $\mathrm{m}^{3} / \mathrm{ano}$, segue que:

$$
V_{k}=\sum_{i=1}^{M} \sum_{j=1}^{N} V T_{i j k} W_{i j}\{k=0,1, \ldots, H-2\}
$$

em que $V T_{i j k}=$ volume total $\left(\mathrm{m}^{3}\right)$ produzido pela i-ésima unidade de manejo assinalada na j-ésima alternativa de 
manejo, no início do período $k ; V_{k}=$ volume total $\left(\mathrm{m}^{3}\right)$ removido no período $k$, representando a demanda a ser atendida no período $K$; e $H=$ horizonte de planejamento.

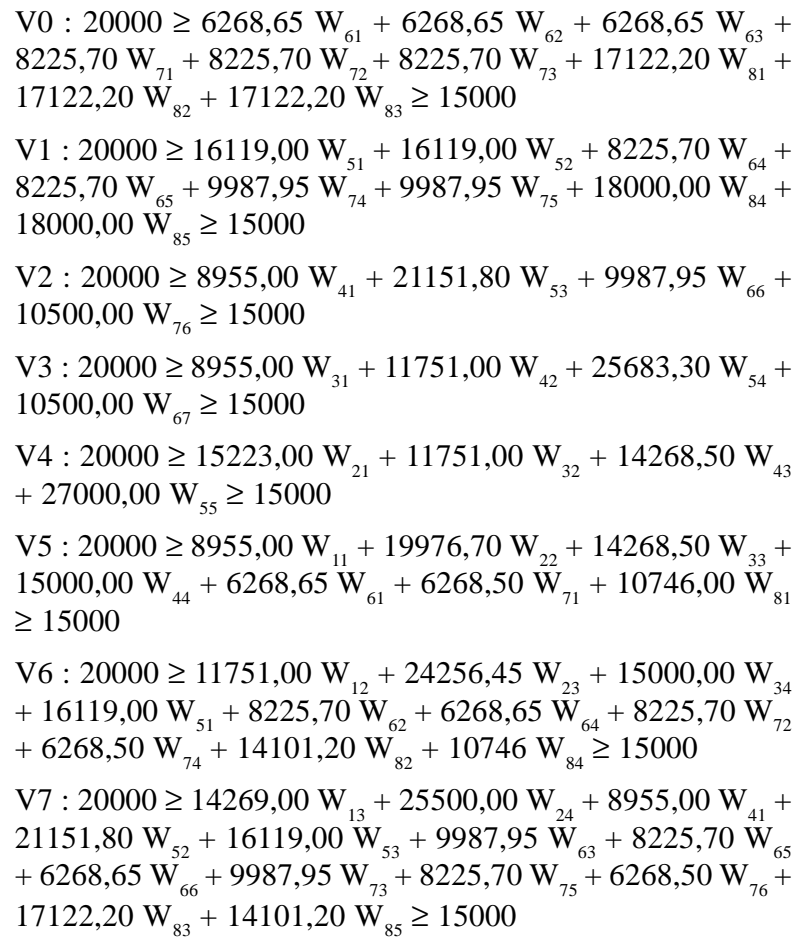

Os modelos de PL e de PI foram resolvidos com o emprego algoritmos Simplex e Branch and Bound, respectivamente, implementados no software LINDO/PC, versão 6.01 (1997). A solução obtida com a PL foi submetida a um arredondamento e, posteriormente, comparada com a solução obtida pelo método de Branch and Baund.

\section{RESULTADOS E DISCUSSÃO}

Para melhor compreensão dos resultados, primeiro é apresentada a resposta para o modelo de PL clássico. Em seguida, é apresentada a resposta para esse mesmo modelo com posterior arredondamento. Finalmente, é apresentada a solução para o problema de acordo com a técnica de Programação Inteira.

\subsection{Solução do modelo de Programação Linear}

A solução do modelo de PL clássico, proposto no item 2.1., responde às questões básicas do planejador florestal, quais sejam: quando, quanto e onde cortar (Quadro 4). Ainda, de acordo com o Quadro 4, pode-se observar que as restrições propostas foram atendidas.

Contudo, o modelo clássico apresenta um inconveniente: o particionamento das unidades de manejo (Quadro 5). Este particionamento ocorre quando mais de uma alternativa é determinada para uma mesma unidade de manejo. Veja, por exemplo, o caso da unidade de manejo 1 , que foi dividida por duas alternativas, quais sejam: cortar 26,11 ha aos 6 anos (variável $\mathrm{X}_{12}{ }^{*}$ ) e cortar 23,89 ha aos 7 anos (variável $\mathrm{X}_{13}{ }^{*}$ ). Desta forma, a unidade de manejo que originalmente possuía 50 ha foi dividida, respectivamente, em 26,11 e 23,89 ha, o que é indesejável do ponto de vista operacional. De acordo com a solução apresentada, das oito unidades de manejo, apenas as unidades 3 e 7 não foram particionadas.

\subsection{Solução do Modelo de Programação Linear com Posterior Arredondamento}

Uma solução prática para o problema descrito no item anterior, utilizada em algumas empresas, consiste no arredondamento das variáveis. Para que a unidade de manejo não seja particionada, pode-se escolher para uma determinada unidade de manejo a alternativa que resultou em maior área. Como exemplo, no caso da unidade de manejo 1, particionada em duas alternativas (Quadro 5), prevaleceria a alternativa de corte aos 6 anos (variável $\mathrm{X}_{12}{ }^{*}$ ) em lugar da alternativa de corte aos 7 anos (variável $\left.\mathrm{X}_{13}{ }^{*}\right)$, uma vez que a área (26,11 ha) destinada para corte aos 6 anos é maior do que a área $(23,89$ ha) destinada para corte aos 7 anos.

Considerando esse novo critério de solução, uma nova solução para o problema é apresentada no Quadro 6 , onde se observam alguns problemas:

- dos oito anos do horizonte de planejamento, em seis as restrições de demanda não foram atendidas;

- nos anos 2, 5 e 7, as produções foram superiores à demanda máxima estabelecida de $20.000 \mathrm{~m}^{3} / \mathrm{ano}$;

- nos anos 1, 3 e 4, as produções encontradas foram inferiores à demanda mínima de $15.000 \mathrm{~m}^{3} / \mathrm{ano}$; e

- o lucro diminuiu.

Esse resultado está de acordo com inferências de Goldbarg e Luna (2000), ou seja, a técnica de arredondamento pode resultar em uma derrocada completa no esforço de modelagem e solução. Os autores afirmam ainda que, com o aumento do número de variáveis do problema, há tendência de que esta situação se agrave cada vez mais.

R. Árvore, Viçosa-MG, v.27, n.5, p.677-688, 2003 
Quadro 4 - Plano de manejo florestal considerando a otimização do lucro, obtido pelo modelo de Programação Linear clássico

Table 4 - Forest management planning considering profit optimization, obtained by Linear Programming

\begin{tabular}{|c|c|c|c|c|}
\hline Ano & Prescrição & Idade de Corte (anos) & Área (ha) & Produção $\left(\mathrm{m}^{3}\right)$ \\
\hline 0 & $\mathrm{X}_{81}$ & 7 & 52,56 & $15.000,00$ \\
\hline Total & - & - & 52,56 & $15.000,00$ \\
\hline 1 & $\mathrm{X}_{64}$ & 6 & 11,83 & $2.780,29$ \\
\hline 1 & $\mathrm{X}_{74}$ & 7 & 35,00 & $9.987,95$ \\
\hline 1 & $\mathrm{X}_{84}$ & 8 & 7,44 & $2.232,00$ \\
\hline Total & - & - & 54,27 & $15.000,00$ \\
\hline 2 & $\mathrm{X}_{53}$ & 6 & 50,43 & $11.852,06$ \\
\hline 2 & $\mathrm{X}_{66}$ & 7 & 23,17 & $6.612,02$ \\
\hline Total & - & - & 73,60 & $18.464,08$ \\
\hline 3 & $\mathrm{X}_{42}$ & 6 & 37,06 & $8.708,84$ \\
\hline 3 & $\mathrm{X}_{54}$ & 7 & 39,57 & $11.292,09$ \\
\hline Total & - & - & $\mathbf{7 6 , 6 3}$ & $20.000,00$ \\
\hline 4 & $\mathrm{X}_{21}$ & 5 & 25,43 & $4.555,51$ \\
\hline 4 & $\mathrm{X}_{32}$ & 6 & 50,00 & $11.752,00$ \\
\hline 4 & $\mathrm{X}_{43}$ & 7 & 12,94 & $3.6292,69$ \\
\hline Total & - & - & 88,37 & $20.000,00$ \\
\hline 5 & $\mathrm{X}_{22}$ & 6 & 45,04 & $10.585,90$ \\
\hline 5 & $\mathrm{X}_{81}$ & 5 & 52,56 & $9.414,50$ \\
\hline Total & - & - & 97,96 & $20.000,00$ \\
\hline 6 & $\mathrm{X}_{12} *$ & 6 & 26,11 & $6.136,47$ \\
\hline 6 & $\mathrm{X}_{23}$ & 7 & 14,52 & $4.143,67$ \\
\hline 6 & $\mathrm{X}_{64}$ & 5 & 11,83 & $2.118,85$ \\
\hline 6 & $\mathrm{X}_{74}$ & 5 & 35,00 & $6.268,50$ \\
\hline 6 & $\mathrm{X}_{84}$ & 5 & 7,44 & $1.232,50$ \\
\hline Total & - & - & 94,90 & $20.000,00$ \\
\hline 7 & $X_{13} *$ & 7 & 23,89 & $6.817,49$ \\
\hline 7 & $X_{53}$ & 5 & 50,43 & $9.032,74$ \\
\hline 7 & $X_{66}$ & 5 & 23,17 & $4.149,75$ \\
\hline Total & - & - & 97,49 & $20.000,00$ \\
\hline
\end{tabular}

Lucro $(\mathrm{R} \$ / \mathrm{ano})=219.582,00$.

\subsection{Resultados do Modelo de Programação Inteira}

A solução do modelo de Programação Inteira é apresentada no Quadro 7. Pode-se observar que as restrições propostas foram atendidas e que apenas uma recomendação de corte obtida no item 3.2. coincidiu com a solução obtida pela PI (variáveis $\mathrm{X}_{81}$ e $\mathrm{W}_{81}$ ), ou seja, das oito unidades de manejo destinadas a corte apenas uma resposta obtida pela PL com posterior arredondamento coincidiu com as obtidas pela PI, mostrando a fragilidade desse método.

R. Árvore, Viçosa-MG, v.27, n.5, p.677-688, 2003
O modelo de PI apresenta também a vantagem de não fracionar as unidades de manejo (Quadro 8). Observa-se, por este quadro, que foi determinada uma única alternativa para cada unidade de manejo. No entanto, quando comparada com a solução do modelo de PL, a solução do problema utilizando PI apresentou redução no valor da função objetivo de $\mathrm{R} \$ 219.582,00$ para $\mathrm{R} \$ 200.988,89$ (aproximadamente 8,47\%), o que é normal, uma vez que a restrição de integridade é uma restrição forte e penaliza o objetivo a ser alcançado (neste caso o de maximização). Também, a resposta com 
arredondamento apresentou maior valor da funçãoobjetivo do que a resposta obtida pela PI, embora esta solução tenha se apresentado inviável para algumas restrições. Cabe lembrar que, do ponto de vista matemático, em hipótese alguma uma solução obtida por PL com posterior arredondamento conseguiria, ao mesmo tempo, atender a todas as restrições e gerar um valor ótimo superior ou inferior, em casos de maximização ou minimização, respectivamente, ao valor ótimo gerado pela PI.

A Figura 1 compara as diferentes soluções de regulação da floresta obtidas pela PL, PL com posterior arredondamento e PI. Por esta figura, pode-se observar que a PL foi o método que proporcionou a menor oscilação entre as produções anuais, sendo estas constantes a partir do terceiro período $\left(20.000 \mathrm{~m}^{3} / \mathrm{ano}\right)$. Cabe salientar que, como o objetivo foi maximizar o lucro, houve tendência de que se produzisse anualmente o máximo volume possível, no caso 20.000 m³/ano. Já para a solução obtida pela PI, a oscilação da produção foi maior, embora tenha atendido aos propósitos inicialmente sugeridos, ou seja, a produção se situou durante todo o horizonte de planejamento entre 15.000 e $20.000 \mathrm{~m}^{3} /$ ano. Considerando a solução obtida por PL com posterior arredondamento, pode-se observar grande oscilação da produção ao longo do horizonte de planejamento. Ainda, o atendimento das restrições de demanda só foi verificado nos períodos 0 e 6 .

Quando se comparou, pela Figura 1, a quantidade de área destinada para corte em cada período, de acordo com as soluções obtidas pelos três métodos, verificou-se resultado muito semelhante ao que foi obtido para a comparação em termos de volume. Este resultado era esperado, uma vez que, conforme definido no item 2 , as unidades de manejo apresentam sítios homogêneos.

É importante mencionar também que, mesmo que apresente a vantagem de determinar uma resposta

Quadro 5 - Alternativas escolhidas para cada unidade de manejo pelo modelo de Programação Linear clássico Table 5 - Alternatives chosen for each management unit by the Linear Programming model

\begin{tabular}{|c|c|c|c|}
\hline Prescrição & Unidade de Manejo & Alternativa & Área (ha) \\
\hline $\mathrm{X}_{12}{ }^{*}$ & 1 & 2 (corte aos 6 anos) & 26,11 \\
\hline \multirow[t]{2}{*}{$X_{13}^{*}$} & 1 & 3 (corte aos 7 anos) & 23,89 \\
\hline & & Área Total & 50,00 \\
\hline $\mathrm{X}_{21}$ & 2 & 1 (corte aos 5 anos) & 25,43 \\
\hline $\mathrm{X}_{22}$ & 2 & 2 (corte aos 6 anos) & 45,04 \\
\hline \multirow[t]{2}{*}{$X_{23}$} & 2 & 3 (corte aos 7 anos) & 14,52 \\
\hline & & Área Total & 85,00 \\
\hline \multirow[t]{2}{*}{$\mathrm{X}_{32}$} & 3 & 2 (corte aos 6 anos) & 50,00 \\
\hline & & Área Total & 50,00 \\
\hline $\mathrm{X}_{42}$ & 4 & 2 (corte aos 6 anos) & 37,06 \\
\hline \multirow[t]{2}{*}{$\mathrm{X}_{43}$} & 4 & 3 (corte aos 7 anos) & 12,94 \\
\hline & & Área Total & 50,00 \\
\hline $\mathrm{X}_{53}$ & 5 & 3 (corte aos 6 e depois aos 5 anos) & 50,43 \\
\hline \multirow[t]{2}{*}{$\mathrm{X}_{54}$} & 5 & 4 (corte aos 7 anos) & 39,57 \\
\hline & & Área Total & 90,00 \\
\hline $\mathrm{X}_{64}$ & 6 & 4 (corte aos 6 e depois aos 5 anos) & 11,83 \\
\hline \multirow[t]{2}{*}{$X_{66}$} & 6 & 6 (corte aos 7 e depois aos 5 anos) & 23,17 \\
\hline & & Área Total & 35,00 \\
\hline \multirow[t]{2}{*}{$X_{74}$} & 7 & 4 (corte aos 7 e depois aos 5 anos) & 35,00 \\
\hline & & Área Total & 35,00 \\
\hline $\mathrm{X}_{81}$ & 8 & 1 (corte aos 7 e depois aos 5 anos) & 52,56 \\
\hline \multirow[t]{2}{*}{$\mathrm{X}_{84}$} & 8 & 4 (corte aos 8 e depois aos 5 anos) & 7,44 \\
\hline & & Área Total & 60,00 \\
\hline
\end{tabular}


operacionalmente mais interessante, o algoritmo de solução utilizado para problemas de PI (normalmente o algoritmo de Branch and Bound) pode se apresentar computacionalmente inviável para problemas de grande porte. Atualmente, problemas com aproximadamente 100 mil variáveis inteiras, dependendo da complexidade

Quadro 6 - Plano de manejo florestal obtido pelo arredondamento da solução do modelo de Programação Linear clássico

Table 6 - Forest management planning obtained by the method of post rounding out optimal solution of the Linear Programming model

\begin{tabular}{|c|c|c|c|c|}
\hline Ano & Prescrição & $\begin{array}{c}\text { Idade de } \\
\text { Corte } \\
\text { (anos) }\end{array}$ & Área (ha) & Produção $\left(\mathrm{m}^{3}\right)$ \\
\hline 0 & $\mathrm{X}_{81}$ & 7 & 60,00 & $17.122,20$ \\
\hline Total & - & - & 60,00 & $17.122,20$ \\
\hline 1 & $\mathrm{X}_{74}$ & 7 & 35,00 & $9.987,95$ \\
\hline Total & - & - & 35,00 & $9.987,95$ \\
\hline 2 & $\mathrm{X}_{53}$ & 6 & 90,00 & $21.151,80$ \\
\hline 2 & $\mathrm{X}_{66}$ & 7 & 35,00 & $9.987,95$ \\
\hline Total & - & - & 125,00 & $31.139,75$ \\
\hline 3 & $\mathrm{X}_{42}$ & 6 & 50,00 & $11.751,00$ \\
\hline Total & - & - & 50,00 & $11.751,00$ \\
\hline 4 & $\mathrm{X}_{32}$ & 6 & 50,00 & $11.751,00$ \\
\hline Total & - & - & 50,00 & $11.751,00$ \\
\hline 5 & $\mathrm{X}_{22}$ & 6 & 85,00 & $19.976,70$ \\
\hline 5 & $X_{81}$ & 5 & 60,00 & $10.746,00$ \\
\hline Total & - & - & 145,00 & $30.722,70$ \\
\hline 6 & $\mathrm{X}_{12}$ & 6 & 50,00 & $11.751,00$ \\
\hline 6 & $X_{74}$ & 5 & 35,00 & $6.268,50$ \\
\hline Total & - & - & 85,00 & $18.019,50$ \\
\hline 7 & $\mathrm{X}_{53}$ & 5 & 90,00 & $16.119,00$ \\
\hline 7 & $\mathrm{X}_{66}$ & 5 & 35,00 & $6.268,50$ \\
\hline Total & - & - & 125,00 & $22.387,50$ \\
\hline
\end{tabular}

Lucro $(\mathrm{R} \$ / \mathrm{ano})=209.578,29$. do modelo e do equipamento de hardware disponível, poderiam ser resolvidos por pacotes comerciais existentes no mercado. Problemas de planejamento florestal que envolvem número de variáveis superior a este demandariam outros métodos para solução, como Algoritmos Genéticos, Busca Tabu e Simulated Anneling.

Quadro 7 - Plano de manejo florestal considerando a otimização do lucro, obtido pela técnica de Programação Inteira

Table 7 - Forest management planning considering profit optimization obtained by the Integer Programming method

\begin{tabular}{|c|c|c|c|c|}
\hline Ano & Prescrição & $\begin{array}{l}\text { Idade de } \\
\text { Corte } \\
\text { (anos) }\end{array}$ & Área (ha) & Produção $\left(\mathrm{m}^{3}\right)$ \\
\hline 0 & $\mathrm{~W}_{81}=1$ & 7 & 60,00 & $17.122,20$ \\
\hline Total & - & - & 60,00 & $17.122,20$ \\
\hline 1 & $\mathrm{~W}_{51}=1$ & 5 & 90,00 & $16.119,00$ \\
\hline Total & - & - & 90,00 & $16.119,00$ \\
\hline 2 & $\mathrm{~W}_{41}=1$ & 5 & 50,00 & $8.955,00$ \\
\hline 2 & $\mathrm{~W}_{76}=1$ & 8 & 35,00 & $10.500,00$ \\
\hline Total & - & - & 85,00 & $19.455,00$ \\
\hline 3 & $\mathrm{~W}_{31}=1$ & 5 & 50,00 & $8.955,00$ \\
\hline 3 & $\mathrm{~W}_{67}=1$ & 8 & 35,00 & $10.500,00$ \\
\hline Total & - & - & 85,00 & $19.455,00$ \\
\hline 4 & $\mathrm{~W}_{21}=1$ & 5 & 85,00 & $15.223,00$ \\
\hline Total & - & - & 85,00 & $15.223,00$ \\
\hline 5 & $\mathrm{~W}_{11}=1$ & 5 & 50,00 & $8.955,00$ \\
\hline 5 & $\mathrm{~W}_{81}=1$ & 5 & 60,00 & $10.746,00$ \\
\hline Total & - & - & 110,00 & $19.701,00$ \\
\hline 6 & $\mathrm{~W}_{51}=1$ & 5 & 90,00 & $16.119,00$ \\
\hline Total & -'"' & - & 90,00 & $16.199,00$ \\
\hline 7 & $\mathrm{~W}_{41}=1$ & 5 & 50,00 & $8.955,00$ \\
\hline 7 & $\mathrm{~W}_{76}=1$ & 5 & 35,00 & $6.268,50$ \\
\hline Total & - & - & 85,00 & $15.223,50$ \\
\hline
\end{tabular}

Lucro $(\mathrm{R} \$ / \mathrm{ano})=200.988,89$.

Quadro 8 - Alternativas escolhidas para cada unidade de manejo pelo modelo de Programação Inteira Table 8 - Alternatives chosen for each management unit by the Integer Programming model

\begin{tabular}{|c|c|c|c|}
\hline Prescrição & Unidade de Manejo & Alternativa & Área (ha) \\
\hline $\mathrm{W}_{11}$ & 1 & 1 (corte aos 5 anos) & 50,00 \\
\hline $\mathrm{W}_{21}$ & 2 & 1 (corte aos 5 anos) & 85,00 \\
\hline $\mathrm{W}_{31}$ & 3 & 1 (corte aos 5 anos) & 50,00 \\
\hline $\mathrm{W}_{41}$ & 4 & 1 (corte aos 5 e depois aos 5 anos) & 50,00 \\
\hline $\mathrm{W}_{51}$ & 5 & 1 (corte aos 5 e depois aos 5 anos) & 90,00 \\
\hline $\mathrm{W}_{67}$ & 6 & 7 (corte aos 8 anos) & 35,00 \\
\hline $\mathrm{W}_{76}$ & 7 & 6 (corte aos 8 e depois aos 5 anos) & 35,00 \\
\hline $\mathrm{W}_{81}$ & 8 & 1 (corte aos 7 e depois aos 5 anos) & 60,00 \\
\hline
\end{tabular}




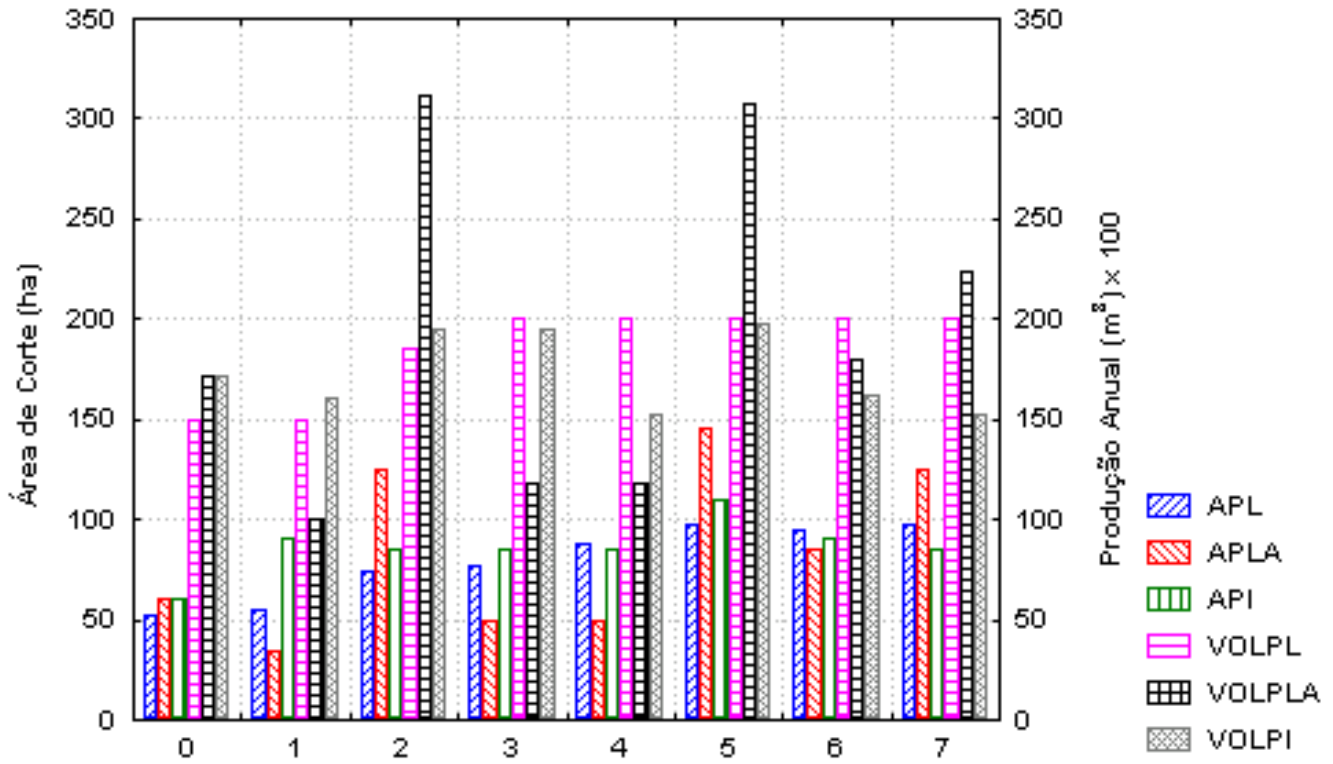

Figura 1 - Comparação entre os métodos de regulação da floresta, em termos de área e volume, em que APL, APLA e API correspondem às áreas anuais cortadas, segundo as soluções obtidas por PL, PL com posterior arredondamento e PI, respectivamente, e VOLPL, VOLPLA e VOLPI correspondem às produções anuais, segundo as soluções obtidas por PL, PL com posterior arredondamento e PI, respectivamente.

Figure 1 - Comparison among the forest regulation methods for area and volume, where APL, APLA and API correspond to the annually-cut areas, according to the solutions obtained by PL, PL with post rounding out and PI, respectively, and VOLPL, VOLPLA and VOLPI corresponding to the annual productions, according to the solutions obtained by PL, PL with post rounding out and $P I$, respectively.

\section{CONCLUSÕES}

Com base nos resultados, pode-se concluir que:

- Do ponto de vista matemático, em problemas de regulação florestal, soluções operacionais resultantes de arredondamentos da solução obtida via PL não devem ser adotadas, pois podem levar ao não-atendimento das restrições, além de não garantirem os objetivos de otimização, quais sejam os de maximizar ou minimizar uma função-objetivo qualquer.

- A solução obtida pela PI atendeu a todas as restrições propostas e resolveu o problema operacional de divisão das unidades de manejo, embora esse método apresente restrições de uso para problemas de grande porte.

\section{REFERÊNCIAS BIBLIOGRÁFICAS}

BOSTON, K.; BETTINGER, P. An analysis of Monte Carlo Integer Programming, Simulated Annealing, and Tabu Search Heuristics for Solving Spatial Harvest Scheduling Problems. Forest Science, v. 45, n. 2, p. 292-301, 1999.
BUONGIORNO, J.; GILLESS, J. K. Forest management and economics - A primer in quantitative methods. Berkeley: 1987. 285 p.

CLUTTER, J. C. et al. Timber management: a quantitative approach. 3.ed. New York: Jonh Willey, 1983. 333 p.

DAVIS, L. S.; JOHNSON, K. N. Forest management. 3.ed. New York: Mc Graw- Hill Book Company, 1987. $790 \mathrm{p}$.

DYKSTRA, D. P. Mathematical programming for natural resource management. New York: McGraw-Hill, 1984. $318 \mathrm{p}$.

GOLDBARG, M. C.; LUNA, H. P. C. Otimização combinatória e programação linear: modelos e algoritmos. Rio de Janeiro: 2000. 649 p.

GOMES, F. S.; MAESTRI, R.; SANQUETA, C. R. Análise financeira de regimes de manejo em povoamentos de Pinus taeda L., visando a produção de madeira para a indústria de papel e celulose. Revista Árvore, v. 22, n. 2, p. 227-243, 1998. 
HILLER, F. S.; LIEBERMAN, G. J. Introduction to operational research. 3.ed. Oakland:, 1980. 829 p.

JOHNSON, K. N.; SCHEURMAN, H. L. Techniques for prescribing optimal timber harvest and investment under different objectives - discussion and synthesis. Forest Science, v. 18, n. 1, p. 1-31, 1977.

LAROZE, A. J. A linear programming, tabu search method for solving forest-level bucking optimization problems. Forest Science, v. 45, n. 1, p. 108-116, 1999.

LAROZE, A. J.; GREBER, B. J. Using Tabu search to generate stand-level, rule-based bucking patterns. Forest Science, v. 43, n. 2, p. 157-169, 1997.

LEITE, H. G. Conversão de troncos em multiprodutos da madeira, utilizando Programação Dinâmica. 1994. 230 f. Tese (Doutorado em Ciência Florestal) - Universidade Federal de Viçosa, Viçosa, 1994.

LOCKWOOD, C.; MOORE, T. Harvest scheduling with spatial constraints: a simulated annealing approach. Canadian Journal Forest Research, v. 23, p. 468-478, 1993.
NOBRE, S. R. A heurística da Razão-R aplicada a problemas de gestão florestal. 1999. 129 f. Dissertação (Mestrado em Administração Rural) - Universidade Federal de Lavras, Lavras: 1999.

REZENDE, J. L. P.; OLIVEIRA, A. D. Avaliação de projetos florestais. Viçosa, MG: Universidade Federal de Viçosa, 1993. 49 p.

RIBEIRO, C. A. A. S. Pesquisa operacional aplicada ao manejo florestal. Viçosa, MG: Universidade Federal de Viçosa, 1996. 107 p. (Notas de aula).

RODRIGUES, F. L. Regulação de florestas eqüiâneas utilizando programação linear. 1997. 109 f. Dissertação (Mestrado em Manejo Florestal) - Universidade Federal de Viçosa, Viçosa, 1997.

RODRIGUES, F. L. Metaheurística e sistema de suporte à decisão no gerenciamento de recursos florestais. 2001. 225 f. Tese (Doutorado em Ciência Florestal) Universidade Federal de Viçosa, Viçosa, 2001.

RODRIGUEZ, L. C. E.; LIMA, A. B. N. P. M. A utilização da Programação Linear na determinação de uma estratégia ótima de reforma de um talhão florestal. IPEF, n. 31, p. 47 53, 1985 